\title{
Technical aspects of trans reduction in modified fats
}

\author{
Gerrit VAN DUIJN \\ TUSCC/Unilever Research, Olivier van Noortlaan \\ 120, 3133 AT Vlaardingen, The Netherlands
}

\begin{abstract}
Modification of the solid phase lines of naturally occurring oils and fats is required to produce optimal hardstocks for fat continuous products like margarines, spreads and shortenings. Trans-free hardstocks are produced by combination of the trans-free modification techniques: full hydrogenation, interesterification, and fractionation together with the choice of the feedstock. The introduction of trans-free hardstocks in domestic margarines since 1995 has drastically reduced the daily trans intake by European consumers.
\end{abstract}

Key words: oil modification, trans fatty acids, full hydrogenation, interesterification, fractionation

sion of dispersed small droplets of an aqueous phase in liquid oil stabilised by a structure of solid fat crystals. These solid fat crystals are important for the following reasons:

- They supply structure, hardness and texture to the product.

- They form a sponge-like structure that prevents the oil from leaking out of the product.

- They avoid coalescence of the dispersed aqueous phase droplets.

To what extend these functionalities are satisfied depends on the amount of crystals present and on the solid fat content of the crystals. The solid fat content as a function of temperature is often referred to as the $\mathrm{N}$-line or solid phase line of the product and is measured using NMR techniques.

The production of fat continuous products with properties adapted to the users needs requires the availability of solid fat crystals (often called hardstocks) with optimised solid phase lines. Margarines and spreads should have a good spreading behaviour when leaving the refrigerator, a good consistency at ambient temperature and a quick oral melt for taste and flavour release. Properties of shortenings are determined by their application.

The range of solid phase lines fulfilling the commonly required criteria for hardstocks for margarine, spread and shortening products is indicated in figure 1.

All commercially traded "natural" oils and fats have solid phase lines outside the optimal range indicated in figure 1. Modification of the solid phase lines of naturally occurring oils and fats is required to produce optimal hardstocks.

The following oil modification techniques are applied:

- Hydrogenation.

- Interesterification.

- Fractionation.

In the following sections these techniques will be reviewed in the context of trans-free hardstock production.

\section{Hydrogenation}

\section{Partial hydrogenation}

Hydrogenation involves the addition of two hydrogen atoms across the unsaturated double bonds $(C=C)$ in the fatty acid groups of the triacylglycerol. The more saturated fat resulting from this reaction has a higher solid phase line than the starting material. Products with different solid phase lines can be obtained by stopping the reaction when only a proportion of the double bonds has been saturated (partial hydrogenation). This is illustrated in figure 2 showing the solid phase lines of palm oil, partially hydrogenated palm oil at two degrees of saturation and (almost) fully hydrogenated palm oil. Compared with the target area of figure 1, palm oil has a too low solid phase line, partially hydrogenated palm oil is within the target area, while fully hydrogenated palm oil has a far too high solid phase line.

Besides saturation, also geometric isomerisation (cis-trans) and positional isomerisation (shift of double bond along the fatty acid chain) will take place during the hydrogenation reaction. These isomerisation reactions are promoted by process conditions like a low hydrogen pressure and a high temperature and the properties of the hydrogenation catalyst (normally nickel on carrier). TFA levels of partially hydrogenated products with industrial Nickel catalyst may vary between 20 and $65 \%$ depending on process conditions and sulphur content of the catalyst [1].

Much lower TFA levels (4-10\%) can be obtained with noble catalyst in the presence of basic additives. Cargill recently launched a low trans partially hydrogenated rapeseed oil with a TFA level of 9\% [2].

TFA also has an influence on the steepness of the solid phase line of partially hydrogenated products. Partially hydrogenated vegetable oil high in TFA has a steeper solid phase line than a product low in TFA. This is illustrated in figure 3, which shows the solid phase lines of "traditionally" hydrogenated rapeseed oil with
Fat continuous products like margarines, spreads, shortenings, etc. consist of an emul- 


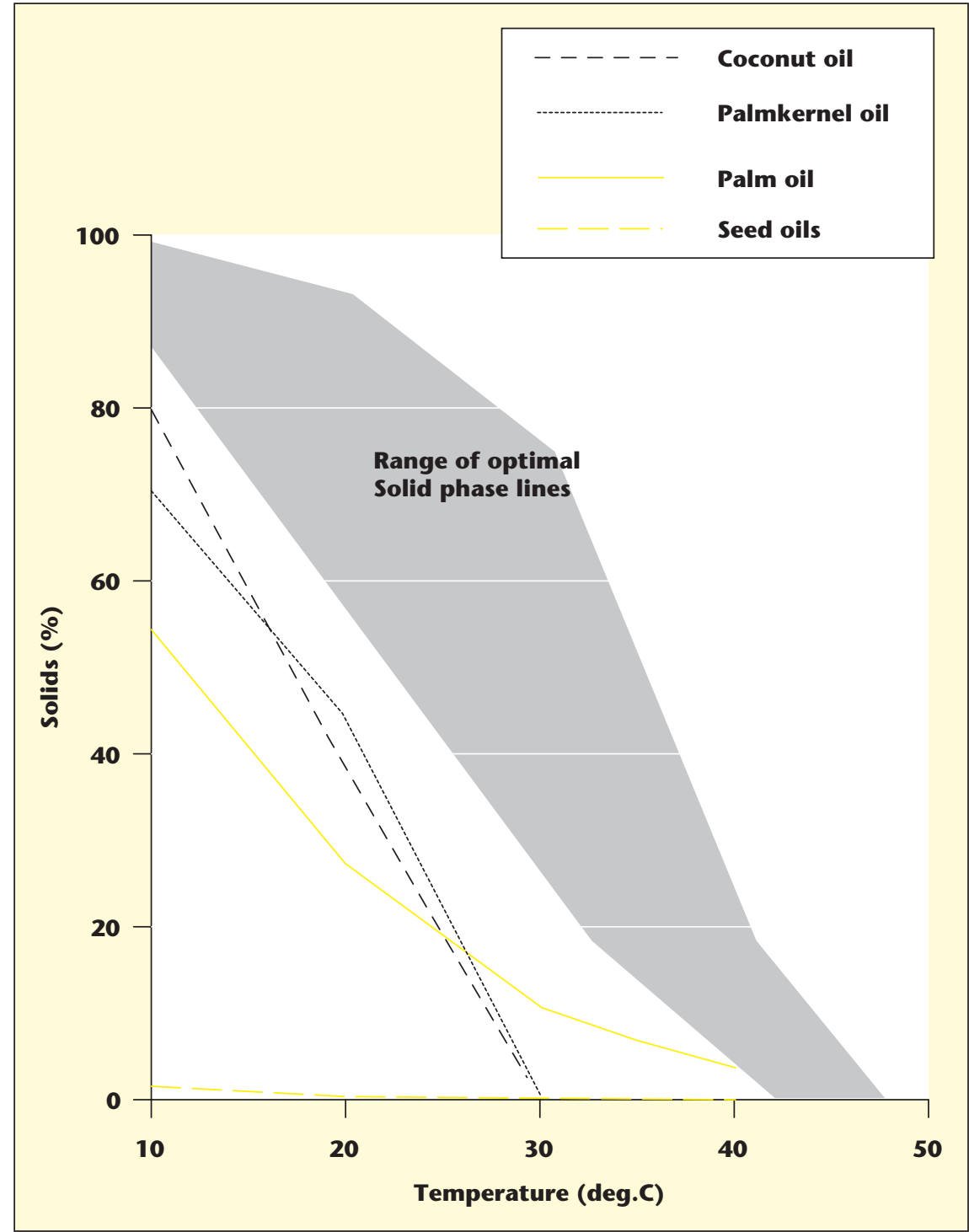

Figure 1. The production of fat continuous products like margarines, shortenings, etc. requires hardstocks with a range of solid phase lines (Grey area). Oils occurring in nature have solid phase lines below this optimal area.

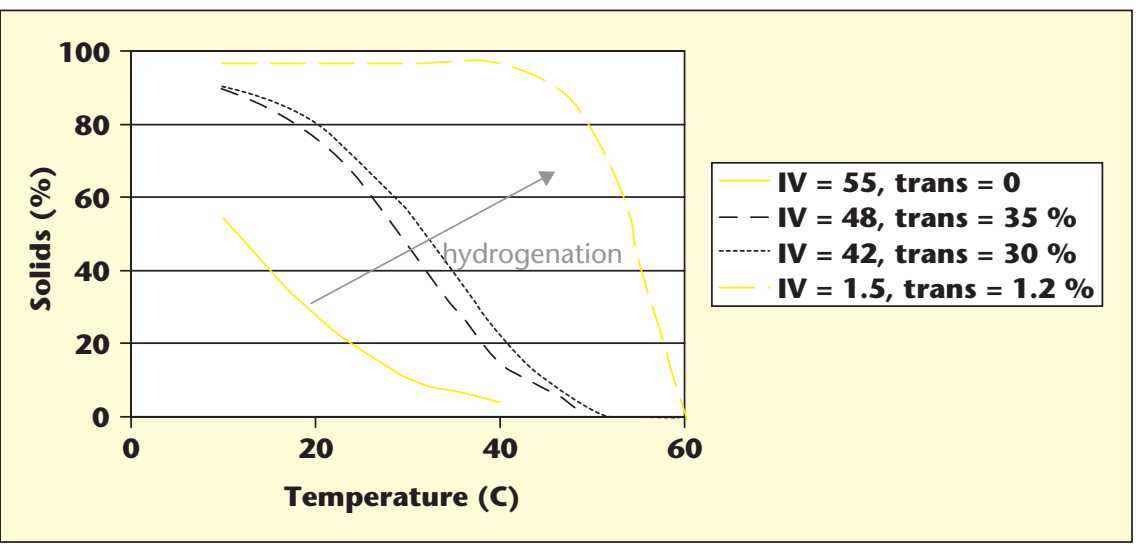

Figure 2. The solid phase lines of palm oil $(I V=55)$, partially hydrogenated palm oil $(I V=42-48)$ and fully hydrogenated palm oil (IV = 1.5). a TFA level of $63 \%$ in comparison with the Cargill Losatra product. The Losatra product has around $20 \%$ solids at $40{ }^{\circ} \mathrm{C}$, while the "traditional" product has no solids at this temperature.

\section{Full hydrogenation}

Complete saturation of all double bonds is practically not achievable in a factory scale hydrogenation process. In practice the residual lodine Value (IV) of a fully hydrogenated product will be around 1-2 (the IV is the measure for the degree of unsaturation of an oil or fat, a completely saturated oil has an IV of zero). A residual IV of 1 corresponds with 1.15\% remaining mono-unsaturated fatty acids in the product. For almost fully hydrogenated fats the thermodynamic equilibrium ratio between cis and trans of 28: 72 is reached. This means that a residual IV of 1 corresponds with TFA level of $0.85 \%$. To obtain a product with a TFA level below $1.25 \%$ (fat requirement for an $80 \%$ fat product with a TFA $<1 \%$ ) the hydrogenation should be continued until an IV below 1.5 is reached (the practical specification of a fully hydrogenated product).

\section{Interesterification}

In interesterification the fatty acids are rearranged on the glycerol fragment of the triacylglycerol molecule. Interesterification is promoted by an alkaline catalyst or by lipase. The most commonly used alkaline catalysts are sodium methylate and sodium ethylate. Rozendaal [3] describes the mechanism of interesterification in detail. Alkaline catalysed interesterifications (e.g. sodium(m)ethylate) produce a product where the fatty acids are randomly distributed over the triacylglycerols and over the three available positions within each molecule. Lipase mainly catalyse rearrangements of the 1 and 3 positions, leaving the 2 position unchanged.

The alkaline catalysed interesterification reaction is very fast; full randomisation is reached within a few minutes after addition of the catalyst. Before catalyst addition, the water and free fatty acid level of the mixture should be low to avoid de-activation of the catalyst. The solid phase line of a random interesterified product is fully determined by the fatty acid composition of the mixture before interesterification. The random distribution of these fatty acids over the triacylglycerol molecules will in general lead to a modification of the solid phase line compared to the solid phase line of the mixture before interesterification. This is illustrated by the following example.

A mixture is prepared of $40 \%$ palmkernel oil and $60 \%$ palm stearin (see section Fractionation). Figure 4 shows the solid phase lines of 


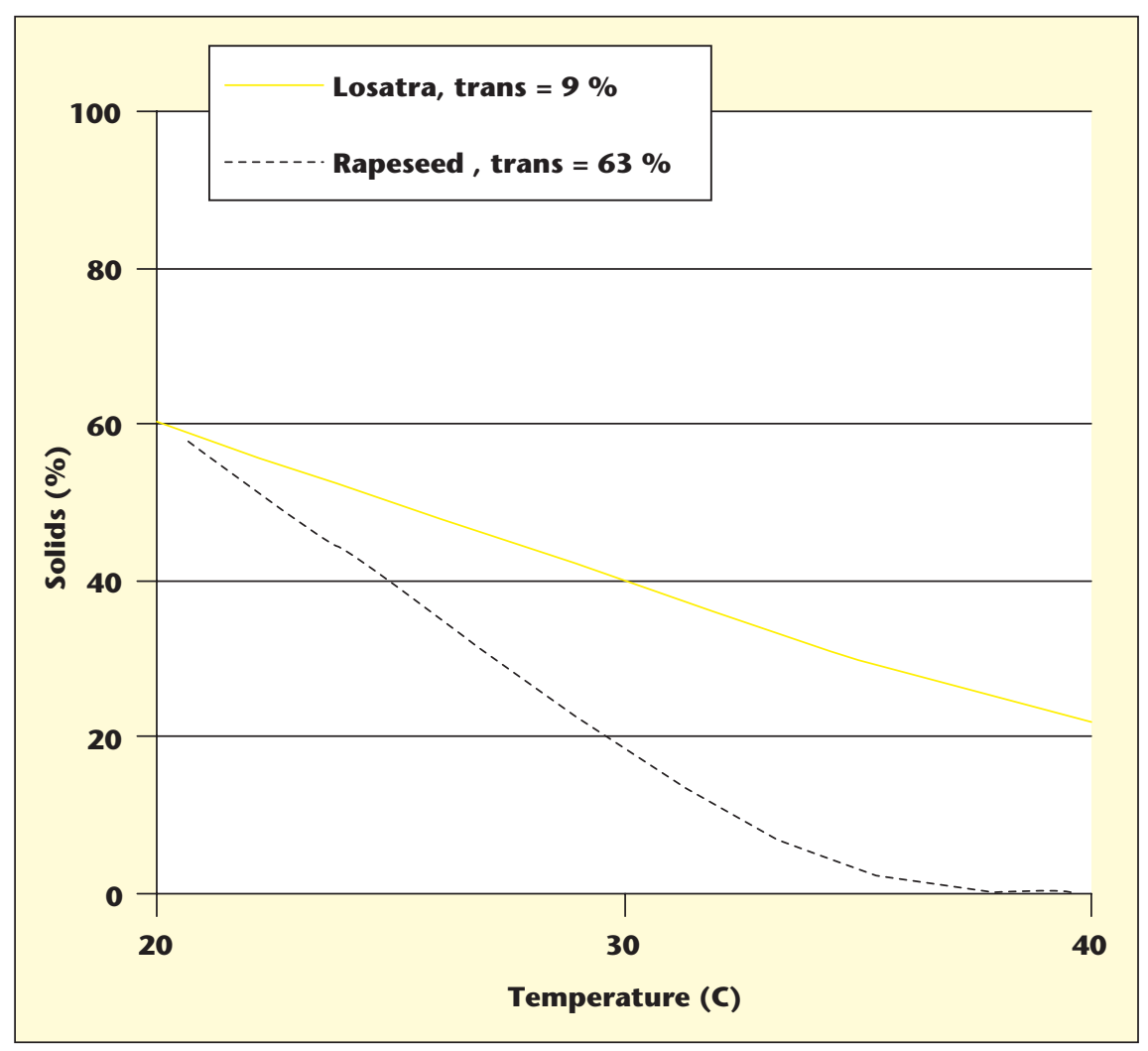

Figure 3. The solid phase lines of low trans partially hydrogenated rapeseed oil (Losatra) and a "classical" high trans hydrogenated rapeseed oil. the two components and of the mixture before interesterification. This mixture has a solid phase level at $40{ }^{\circ} \mathrm{C}$ of around $15 \%$, which is too high for a good oral melt performance. After random interesterification this solid phase value drops to almost zero, making this interesterified mixture better suited as hardstock for fat continuous products.

\section{Fractionation}

Fractionation is the controlled crystallisation of the more saturated and/or longer chain triacylglycerols, followed by separation of the solid phase (named stearin) and liquid phase (named olein). By far the most important oil, fractionated world wide, is palm oil, the main

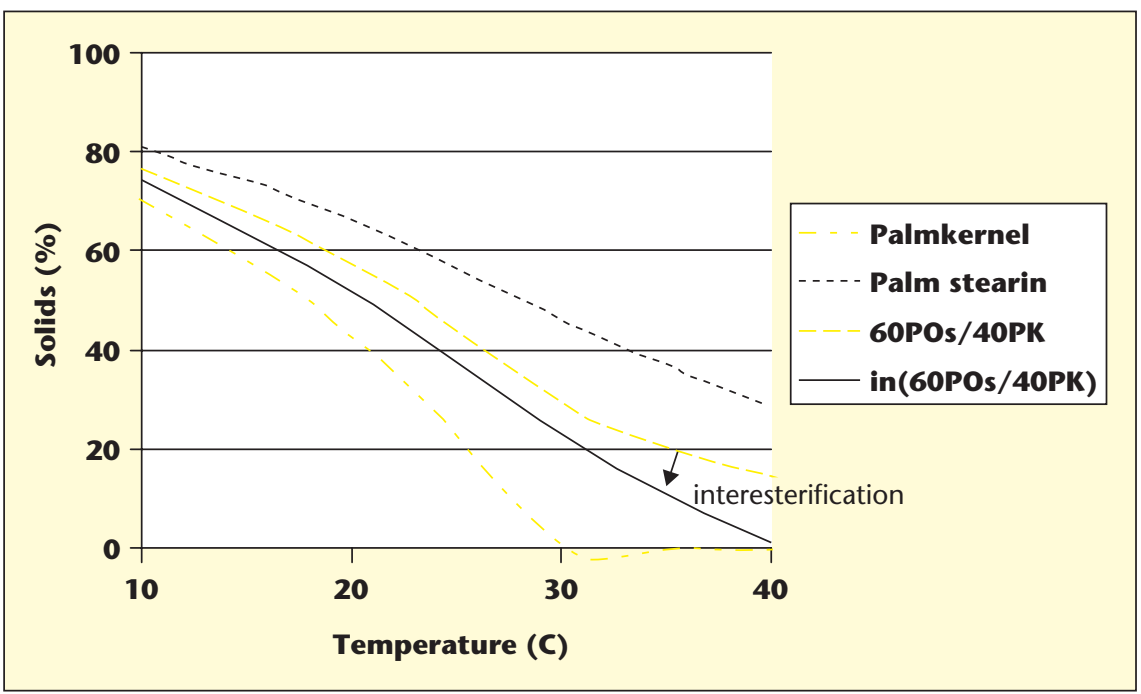

Figure 4. The solid phase lines of: palm stearin and palmkernel oil, the mixture of these two oils, and this mixture after interesterification. reason being the demand for clear liquid oil (palm olein). The solid product of palm oil fractionation (palm stearin) is for a long time used as feedstock for the production of cocoa butter equivalents/replacers and for soap manufacturing. More recent is the use as hardstock for fat continuous products, this requires sometimes a second fractionation of the stearin.

The aim for the fractionation process is to grow large, dense crystal agglomerates that can easily be separated from the liquid oil by filtration. Crystal nuclei are formed when oil is cooled to below its saturation level. These crystal nuclei will grow to larger crystals driven by the supersaturation of the slurry. The crystals will combine into fragile crystal agglomerates. Attrition, due to shear stresses in the stirred crystalliser and during transfer to the filter, will break these agglomerates in smaller pieces. Most modern fractionation plants use membrane filterpresses; these enable the filter cake (produced by simple filtration) to be squeezed to both increase the olein yield and produce a harder stearin.

Both the olein and the stearin may be fractionated a second time. This produces 4 new fractions (figure 5), a "Super Olein" as a liquid cooking oil, two mid fraction with solid phase lines close to palm oil and the high solids fraction "Super Stearin" [4]. Further fractionation of the "Soft Mid Fraction" produces the "Hard Mid Fraction" and the "Mid Olein". The "Hard Mid Fraction" has a steep solid phase line in the temperature range 20 to $30^{\circ} \mathrm{C}$ (figure 6), this may be interesting for specific applications. The "Super Stearin" has high solid values in the complete temperature range from 10 to $40^{\circ} \mathrm{C}$, interesterification with a more unsaturated or shorter chain length oil will be required to reduce the solid values at the higher temperatures.

\section{Trans-free and low-trans hardstocks}

The combination of the trans-free modification techniques full hydrogenation, interesterification and fractionation together with the choice of the feedstock can be used to produce virtually trans-free hardstocks with a range of physical properties.

With respect to the solid phase line, the following principles should be considered:

- Hydrogenation is the only modification technique that can create solids. Hardstock production from liquid seed oils should therefore always start with hydrogenation, for trans-free hardstocks this must be full hydrogenation $(\mathrm{IV}<1.25)$. The product obtained by full hydrogenation is too high in solids for direct use as hardstock and should be interesterified with liquid oil to reduce the solids level. 


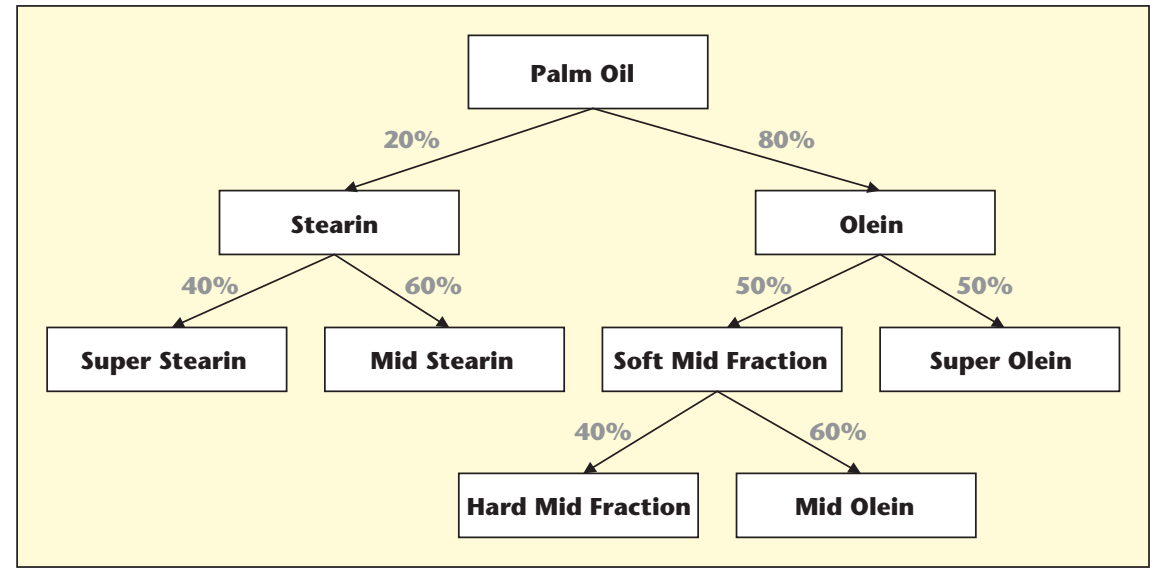

Figure 5. Products from palm oil fractionation.

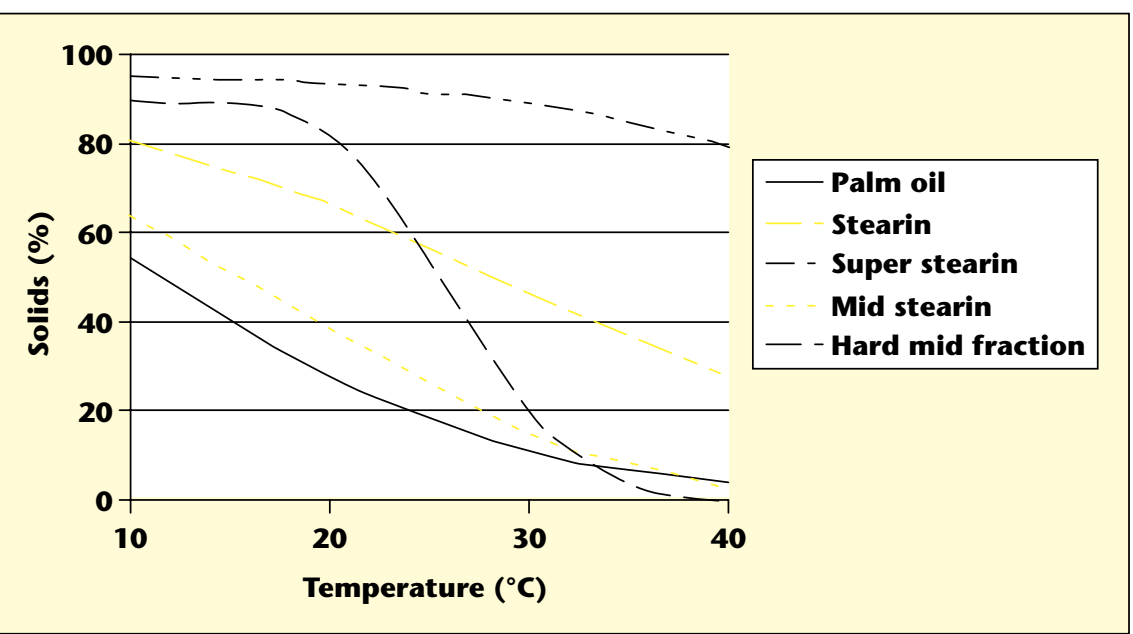

Figure 6. Solid phase lines of the "hard" palm oil fractions.

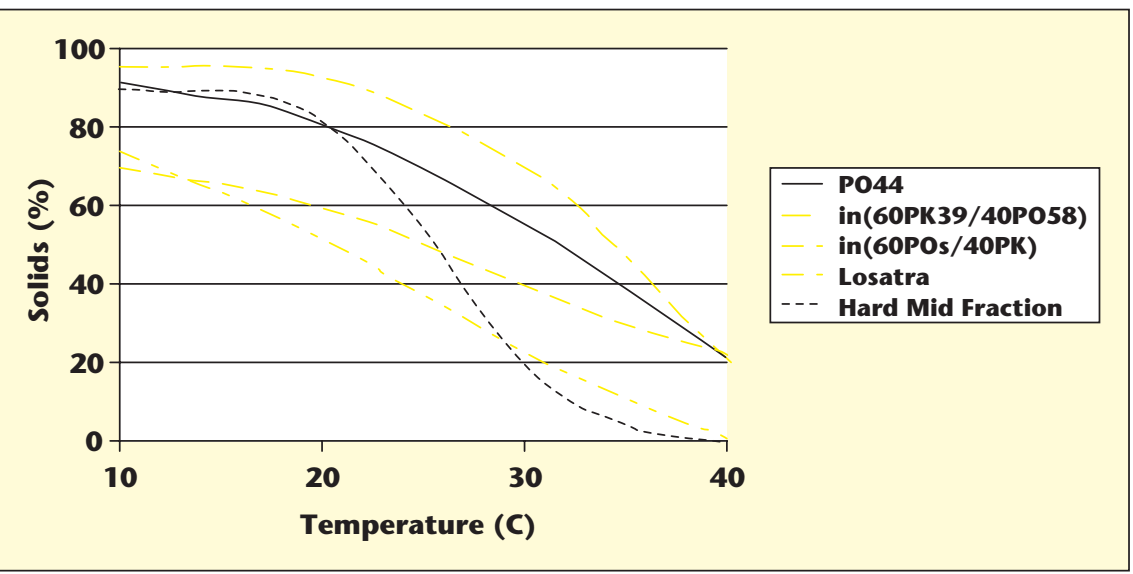

Figure 7. Solid phase lines of examples of trans-free and low-trans hardstocks.

These lines are compared with the solid phase line of high trans partially hydrogenated palm oil (PO44).
- Low trans hardstocks can be produced from liquid seed oils by partial hydrogenation using a special process and catalyst (Losatra from Cargill). These products still contain up to $10 \%$ trans and have a reduced steepness of the solid phase line. The level of saturated fatty acids is relatively low compared to trans-free hardstocks (the saturated fatty acid level of Losatra is $45 \%)$.

- Interesterification will randomly redistribute the fatty acids of the feedstocks over the triacylglycerol molecules. This technique can be used to create triacylglycerols with mixed saturated and unsaturated fatty acid chains starting e.g. from a mixture of a fully hydrogenated and a non hydrogenated liquid seed oil. It can also be used to create triacylglycerols with mixed short and long chain fatty acids starting from e.g. palm stearin and palmkernel or coconut oil.

- Fractionation will split the feedstock in a high melting and a low melting fraction. Single fractionation of palm oil may not be enough to generate sufficient solids. In that case double fractionation can be applied.

Figure 7 shows the solid phase lines of hardstocks produced with the mentioned techniques. As reference, also partially hydrogenated palm oil with a high trans level (PO44, with melting point $44{ }^{\circ} \mathrm{C}$ and $35 \%$ TFA) is shown. The other products are:

- Losatra,

- Interesterified mixture of $60 \%$ palm stearin and $40 \%$ palmkernel oil (in(60POs/40PK).

- Interesterified mixture of $40 \%$ fully hydrogenated palm oil and $60 \%$ fully hydrogenated palmkernel oil (in(60PK39/40PO58) [1].

- The "Hard Mid Fraction" produced by double fractionation.

It should be noted that also other physical parameters than solid phase line alone (like rate of crystallisation) determine the successful application of a hardstock in a product. An overview of these parameters is recently given by Flőter and van Duijn [5].

\section{The results of the industrial project}

In 1995, Unilever decided to practically eliminate trans containing components from the margarine compositions in Europe. The European margarine manufacturers followed this decision. The transfer from margarine production based on high trans partially hydrogenated fats to virtually trans-free components has been established in a relative short period [1]. The effect on daily trans intake by European consumers is impressive. This can best be illustrated by the reduction of the trans fatty acid 
volume in domestic margarines sold by Unilever in Europe. In 1994, just before the decision to eliminate TFA, Unilever sold 130,000 tons TFA in their domestic margarines. In 2002, this figure has been reduced to below 10,000 tons. A similar reduction has been achieved in Africa and Asia. The strong dependence on partially hydrogenated soybean oil has delayed the trans reduction in North and South
America. This will change due to the compulsory labelling of trans fat in the USA.

\section{REFERENCES}

1. VAN DUIJN G. Technical aspects of trans reduction in margarines. OCL 2000; 7: 95-8.

2. CARGILL. Technical specifications Losatra 10 . Cargill refined Oils Europe, 2004, www.losatra .com.
3. ROZENDAALA, MACREA AR. Interesterification of Oils and Fats. In: Gunstone FD, Padley FB, eds. Lipid Technologies and Applications. New York: Marcel Dekker, 1997: 223-63.

4. AFECG. DeSmet presentation on Fractionation. Paris: AFECG, 2005.

5. FLÖTER E, VAN DUIJN G. Trans-free fats. In: FD. Gunstone (Ed.), Modifying Lipids for use in foods, chapter 17, Woodhead publishing (to be published). 